\title{
Fourteen Limit Cycles in a Seven-Degree Nilpotent System
}

\author{
Wentao Huang, ${ }^{1,2}$ Ting Chen, ${ }^{1}$ and Tianlong $\mathbf{G u}^{1}$ \\ ${ }^{1}$ Guangxi Key Laboratory of Trusted Software, School of Computing Science and Mathematics, \\ Guilin University of Electronic Technology, Guilin 541004, China \\ ${ }^{2}$ Department of Mathematics, Hezhou University, Hezhou 542800, China
}

Correspondence should be addressed to Wentao Huang; huangwentao@163.com

Received 13 August 2013; Accepted 30 October 2013

Academic Editor: Isaac Garcia

Copyright (C) 2013 Wentao Huang et al. This is an open access article distributed under the Creative Commons Attribution License, which permits unrestricted use, distribution, and reproduction in any medium, provided the original work is properly cited.

Center conditions and the bifurcation of limit cycles for a seven-degree polynomial differential system in which the origin is a nilpotent critical point are studied. Using the computer algebra system Mathematica, the first 14 quasi-Lyapunov constants of the origin are obtained, and then the conditions for the origin to be a center and the 14th-order fine focus are derived, respectively. Finally, we prove that the system has 14 limit cycles bifurcated from the origin under a small perturbation. As far as we know, this is the first example of a seven-degree system with 14 limit cycles bifurcated from a nilpotent critical point.

\section{Introduction}

In the qualitative theory of planar differential equations, the center-focus problem and bifurcation of limit cycles for nilpotent system

$$
\begin{gathered}
\frac{d x}{d t}=y+\sum_{k+j=2}^{\infty} a_{k j} x^{k} y^{j}=X(x, y), \\
\frac{d y}{d t}=\sum_{k+j=2}^{\infty} b_{k j} x^{k} y^{j}=Y(x, y),
\end{gathered}
$$

are known as a difficult problem. Some advance of this problem can be dated back to [1-3]. In recent years, due to the improvement of research method and development of computer symbolic computation, the problem has attracted more and more scholars' attention and has received a lot of results. For instance, in $[4,5]$, the center conditions of the nilpotent critical points were obtained for several systems. In [6] the center conditions and the bifurcations of limit cycles were investigated for a quintic and a nine-degree nilpotent systems. The center and the limit cycles problems of a quintic nilpotent system were also solved in [7]. And in [8], the authors gave a recursive method to calculate quasi-Lyapunov constants of the nilpotent critical point. The nilpotent center problem and limit cycles bifurcations were performed also in
[9]. It is interesting how many limit cycles can be bifurcated from the nilpotent critical point. Let $N(n)$ be the maximum possible number of limit cycles bifurcated from a nilpotent critical point of system (1) when $X$ and $Y$ are of degree at most $n$. The known results of $N(n)$ are: Andreev et al. given have $N(3) \geq 2, N(5) \geq 5, N(7) \geq 9$, see [5]. Y. Liu and J. Li showed $N(3) \geq 4, N(3) \geq 7, N(3) \geq 8$, see $[8,10-12]$. Li et al. found $N(7) \geq 12$ in [13]. Recently, Li et al. [14] obtained $N(7) \geq 13$.

In this paper, we study the bifurcation of limit cycles for a seven-degree nilpotent system with the following form:

$$
\begin{aligned}
\frac{d x}{d t}= & \delta x+y+a_{30} x^{3}+a_{12} x y^{2}+a_{32} x^{3} y^{2}+a_{14} x y^{4} \\
& +a_{05} y^{5}+a_{06} y^{6}+a_{15} x y^{5}+a_{24} x^{2} y^{4}+a_{33} x^{3} y^{3} \\
& +a_{51} x^{5} y+a_{07} y^{7}+a_{16} x y^{6}+a_{25} x^{2} y^{5} \\
& +a_{34} x^{3} y^{4}+a_{43} x^{4} y^{3}+a_{61} x^{6} y, \\
\frac{d y}{d t}= & 2 \delta y-2 x^{3}+x y^{2}+b_{33} x^{3} y^{3}+a_{51} x^{4} y^{2} .
\end{aligned}
$$

By the computation of the quasi-Lyapunov constants, we prove that its perturbed system has 14 small-amplitude limit cycles bifurcated from the origin, namely, $N(7) \geq 14$ which improves the result in [14]. 
In Section 2, we give some preliminary knowledge concerning the nilpotent critical point. In Section 3, we obtain the first 14 quasi-Lyapunov constants and derive the sufficient and necessary conditions of the origin to be a center and a 14 th-order fine focus. At the end, it is proved that there exist 14 limit cycles in the neighborhood of the origin of the system.

\section{Focal Values and Quasi-Lyapunov Constants}

In order to discuss limit cycles of the system, we state some preliminary results given by [8].

According to [2], the origin of system is a 3th-order monodromic critical point and a center or a focus if and only if $b_{20}=0,\left(2 a_{20}-b_{11}\right)^{2}+8 b_{30} \leq 0$. Without loss of generality, we assume that $a_{20}=\mu, b_{20}=0, b_{11}=2 \mu, b_{30}=-2$, otherwise let $\left(2 a_{20}-b_{11}\right)^{2}+8 b_{30}=-16 \lambda^{2}, 2 a_{20}+b_{11}=4 \lambda \mu$.

Under the substitutions

$$
\eta=\lambda y+\frac{1}{4}\left(2 a_{20}-b_{11}\right)^{2} \lambda x^{2} \quad \xi=\lambda x
$$

system (1) becomes

$$
\begin{gathered}
\frac{d x}{d t}=y+\mu x^{2}+\sum_{k+2 j=3}^{\infty} a_{k j} x^{k} y^{j}=X(x, y), \\
\frac{d y}{d t}=-2 x^{3}+2 \mu x y+\sum_{k+2 j=4}^{\infty} b_{k j} x^{k} y^{j}=Y(x, y) .
\end{gathered}
$$
nates,

By the transformation of the generalized polar coordi-

$$
x=r \cos \theta \quad y=r^{2} \cos \theta
$$

system (4) is transformed into

$$
\frac{d r}{d \theta}=\frac{\cos \theta R_{1}(\theta)}{Q_{1}(\theta)}+o(r)
$$

where

$$
\begin{gathered}
R_{1}(\theta)=\sin \theta\left(1-2 \cos ^{2} \theta\right)+\mu\left(\cos ^{2} \theta+2 \sin ^{2} \theta\right), \\
Q_{1}(\theta)=-2\left(\cos ^{4} \theta+\sin ^{2} \theta\right)<0 .
\end{gathered}
$$

For sufficiently small $h$, let

$$
r=\widetilde{r}(\theta, h)=\sum_{k=1}^{\infty} v_{k}(\theta) h^{k}
$$

be a solution of (6) satisfying the initial value condition $\left.r\right|_{\theta=0}=h$, where

$$
\begin{gathered}
v_{1}(\theta)=\left(\cos ^{4} \theta+\sin ^{2} \theta\right)^{-1 / 4} \\
\quad \times \exp \left(\left(\frac{-\mu}{2}\right) \arctan \left(\frac{\sin \theta}{\cos ^{2} \theta}\right)\right), \\
v_{1}(k \pi)=1, \quad k=0, \pm 1, \pm 2 \ldots
\end{gathered}
$$

Because for all sufficiently small $r$, there is $d \theta / d t<0$, in a small neighborhood; we obtain the Poincaré return map of (6) in a small neighborhood of the origin as follows:

$$
\Delta(h)=\tilde{r}(-2 \pi, h)-h=\sum_{k=2}^{\infty} v_{k}(-2 \pi) h^{k} .
$$

Lemma 1. For any positive integer $m, v_{2 m+1}(-2 \pi)$ has the form

$$
\nu_{2 m+1}(-2 \pi)=\sum_{k=1}^{\infty} \zeta_{m}^{(k)} \nu_{2 k}(-2 \pi)
$$

where $\zeta_{m}^{(k)}$ is a polynomial of $v_{i}(\pi), v_{i}(2 \pi), v_{i}(-2 \pi),(i=$ $2,3, \ldots 2 m)$ with rational coefficients.

Definition 2. (i) For any positive integer $m, v_{2 m}(-2 \pi)$ is called the $m$ th-order focal value of system (4) at the origin; (ii) if $\nu_{2}(-2 \pi) \neq 0$, the origin of system (4) is called an 1th-order weak focus; if there is an integer $m>1$ such that $\nu_{2}(-2 \pi)=$ $\nu_{4}(-2 \pi)=\cdots=v_{2 m-2}(-2 \pi)=0, \nu_{2 m}(-2 \pi) \neq 0$, then the origin of system (4) is called a $m$ th-order weak focus; (iii) if for all positive integer $m$, we have $\nu_{2 m}(-2 \pi)=0$, the origin of system (4) is called a center.

Lemma 3. For system (4), one can derive successively the formal series

$$
M(x, y)=x^{4}+y^{2}+o\left(r^{4}\right)
$$

such that

$$
\begin{gathered}
\left(\frac{\partial X}{\partial x}+\frac{\partial Y}{\partial y}\right) M-(s+1)\left(\frac{\partial M}{\partial x} X+\frac{\partial M}{\partial y} Y\right) \\
=\sum_{m=1}^{\infty} \lambda_{m}\left[(2 m-4 s-1) x^{2 m+4}+o\left(r^{2 m+4}\right)\right] .
\end{gathered}
$$

Lemma 4. If there exists a natural number s and formal series

$$
M(x, y)=x^{4}+y^{2}+o\left(r^{4}\right)
$$

such that (13) holds, then

$$
v_{2 m}(-2 \pi) \sim \sigma_{m} \lambda_{m}, \quad m=1,2,3 \ldots,
$$

where

$$
\begin{aligned}
\sigma_{m}=\frac{1}{2} \int_{0}^{2 \pi} & \left(1+\sin ^{2} \theta\right) \cos ^{2 m+4} \theta \\
& \times\left(\left(\sin ^{4} \theta+\sin ^{2} \theta\right)^{2 m+7 / 4}\right. \\
& \left.\quad \times \exp \left(\left(2 m-\frac{1}{2}\right) \mu \arctan \frac{\sin \theta}{\cos \theta}\right)\right)^{-1} d \theta>0 .
\end{aligned}
$$

In (15), is the symbol of algebraic equivalence, meaning that there exists $\xi_{m}^{(k)}(k=1,2, \ldots m-1)$, polynomial functions of the coefficients of system (4), such that

$$
\nu_{2 m+1}(-2 \pi)=\sigma_{m} \lambda_{m}+\sum_{k=1}^{m-1} \xi_{m}^{(k)} \lambda_{k} .
$$


Definition 5. In Lemma $4, \lambda_{m}$ is called the $m$ th-order quasiLyapunov constant of the origin of system (4).

Lemma 6. For system (4), one can derive successively the formal series

$$
M(x, y)=y^{2}+\sum_{\alpha+\beta=3}^{\infty} c_{\alpha \beta} x^{\alpha} y^{\beta}
$$

such that

$$
\begin{gathered}
\left(\frac{\partial X}{\partial x}+\frac{\partial Y}{\partial y}\right) M-(s+1)\left(\frac{\partial M}{\partial x} X+\frac{\partial M}{\partial y} Y\right) \\
=\sum_{m=3}^{\infty} \omega_{m}(s, \mu) x^{m},
\end{gathered}
$$

where $c_{00}=c_{10}=c_{01}=c_{20}=c_{11}=0, c_{02}=1$. For $\alpha \geq 1$, $\alpha+\beta \geq 3, c_{\alpha \beta}$, and $\omega_{m}(s, \mu)$ are determined by the following recursive formulas:

$$
\begin{gathered}
c_{\alpha \beta}=\frac{1}{(s+1) \alpha}\left(A_{\alpha-1, \beta+1}+B_{\alpha-1, \beta+1}\right), \\
\omega_{m}(s, \mu)=A_{m, 0}+B_{m, 0},
\end{gathered}
$$

where

$$
\begin{aligned}
& A_{\alpha \beta}=\sum_{k+j=2}^{\alpha+\beta-1}[k-(s+1)(\alpha-k+1)] a_{k j} c_{\alpha-k+1, \beta-j}, \\
& B_{\alpha \beta}=\sum_{k+j=2}^{\alpha+\beta-1}[j-(s+1)(\beta-j+1)] b_{k j} c_{\alpha-k, \beta-j+1} .
\end{aligned}
$$

By choosing $\left\{c_{0 \beta}\right\}$ such that

$$
\omega_{2 k+1}(s, \mu)=0, \quad k=1,2, \ldots,
$$

one has

$$
\lambda_{m}=\frac{\omega_{2 m+4}(s, \mu)}{2 m-4 s-1} .
$$

One considers the perturbed system of system (4)

$$
\begin{gathered}
\frac{d x}{d t}=\delta x+y+\mu x^{2}+\sum_{k+2 j=3}^{\infty} a_{k j} x^{k} y^{j}, \\
\frac{d y}{d t}=2 \delta y-2 x^{3}+2 \mu x y+\sum_{k+2 j=4}^{\infty} b_{k j} x^{k} y^{j} .
\end{gathered}
$$

For system $\left.(24)\right|_{\delta=0}$, from Lemma 4 , we know that the first nonvanishing quasi-Lyapunov constant $\lambda_{m}$ is positive constant times as much as the first nonvanishing focal value, so the former shows the same effect as the latter in the study of bifurcation of limit cycles. From [10, Theorem 4.7], we have the following.

Theorem 7. For the system (27) $\left.\right|_{\delta=0}$, assume that the quasiLyapunov constants of the origin $\lambda_{i}(i=1,2, \ldots)$ have $k$ independent parameters $\gamma=\left(\gamma_{1}, \gamma_{2}, \ldots, \gamma_{k}\right)$; that is, $\lambda_{i}=$ $\lambda_{i}\left(\gamma_{1}, \gamma_{2}, \ldots, \gamma_{k}\right)$. If $\gamma=\gamma_{0}$, the origin of the system (4) is an $m$ th-order weak focus $(m \leq k)$, and the Jacobian determinant

$$
\left.\frac{\partial\left(\lambda_{1}, \lambda_{2}, \ldots, \lambda_{m-1}\right)}{\partial\left(\gamma_{1}, \gamma_{2}, \ldots, \gamma_{m-1}\right)}\right|_{\gamma=\gamma_{0}} \neq 0
$$

then, the perturbed system (24) exists $m$ small amplitude limit cycles bifurcated from the origin.

\section{Criterion of Center Focus and Bifurcation of Limit Cycles}

Applying the recursive formulas in Lemma 6, we compute the quasi-Lyapunov constants of the origin of system (2) $\left.\right|_{\delta=0}$ with the computer algebra system Mathematica and obtain the following result.

Theorem 8. For system (2)| $\left.\right|_{\delta=0}$, the first 14 quasi-Lyapunov constants are as follows:

$$
\begin{aligned}
& \lambda_{1}=a_{30} \text {, } \\
& \lambda_{2}=\frac{2}{5} a_{12} \text {, } \\
& \lambda_{3}=\frac{2}{7} a_{32} \text {, } \\
& \lambda_{4}=\frac{4}{15} a_{14}, \\
& \lambda_{5}=\frac{12}{77} a_{34}, \\
& \lambda_{6}=\frac{2}{195}\left(20 a_{16}+3 a_{51} b_{33}\right) \text {, } \\
& \lambda_{7}=\frac{1}{385} b_{33}\left(35 a_{51}-8 a_{33}\right) \text {, } \\
& \lambda_{8}=\frac{7}{13260} b_{33}\left(128 a_{15}-355 a_{51}\right) \text {, } \\
& \lambda_{9}=\frac{3}{33440} b_{33} a_{51}\left(1385+64 a_{61}\right) \text {, } \\
& \lambda_{10}=\frac{1}{278460} b_{33} a_{51} \\
& \times\left(-192495+12320 a_{05}+1904 a_{43}\right), \\
& \lambda_{11}=\frac{9}{1184444800} b_{33} a_{51} \\
& \times\left(317763455+1688064 a_{43}+1158080 a_{51}^{2}\right), \\
& \lambda_{12}=\frac{1}{505504614521088000} b_{33} a_{51} \\
& \times\left(424870735079675775-8480461063976518 a_{51}^{2}\right. \\
& \left.-164955456258816 b_{33}^{2}\right),
\end{aligned}
$$




$$
\begin{aligned}
\lambda_{13}= & \frac{1}{2497759223828804812800} \\
& \times b_{33} a_{51}(1154557205782671354192175 \\
\lambda_{14}= & -\frac{1}{1926846314779614102444810240000} b_{33} a_{51} \\
& \times(1913839774991447312487020909964625 \\
& \quad-386160437769555260227746202006848 a_{51}^{2} \\
& \left.\quad+457974511144735287048192000 a_{51}^{4}\right) .
\end{aligned}
$$

Here, every $\lambda_{k}(k=1,2, \ldots, 14)$ was computed under the assumption $\lambda_{1}=\lambda_{2}=\cdots=\lambda_{k-1}=0$.

It is easy to obtain the following Theorem.

Theorem 9. For system (2) $\left.\right|_{\delta=0}$, the first 14 quasi-Lyapunov constants at the origin are all zero if and only if the following condition is satisfied:

$$
a_{30}=a_{12}=a_{32}=a_{14}=a_{34}=a_{51}=a_{33}=a_{15}=a_{16}=0 .
$$

If $\delta=0$ and the condition (27) holds, system (2) becomes

$$
\begin{aligned}
\frac{d x}{d t}=y & +a_{05} y^{5}+a_{06} y^{6}+a_{24} x^{2} y^{4} \\
& +a_{07} y^{7}+a_{25} x^{2} y^{5}+a_{43} x^{4} y^{3}+a_{61} x^{6} y, \\
& \frac{d y}{d t}=-2 x^{3}+x y^{2}+b_{33} x^{3} y^{3},
\end{aligned}
$$

$$
\begin{aligned}
& \left.\frac{\partial\left(\lambda_{1}, \lambda_{2}, \lambda_{3}, \lambda_{4}, \lambda_{5}, \lambda_{6}, \lambda_{7}, \lambda_{8}, \lambda_{9}, \lambda_{10}, \lambda_{11}, \lambda_{12}, \lambda_{13}\right)}{\partial\left(a_{30}, a_{12}, a_{32}, a_{14}, a_{34}, a_{16}, a_{33}, a_{15}, a_{61}, a_{05}, a_{43}, a_{51}, b_{33}\right)}\right|_{(29)} \\
& \quad=-\frac{11259131158497337756164795883686035195310097999201613627491381814272 a_{51}^{4} b_{33}^{6}}{110636634525265639383282317978327920684865639296808353136757452754003615234375} \\
& \quad \approx-2526.4563514134 \neq 0 .
\end{aligned}
$$

From (30) and Theorem 7, one has the following.

Theorem 12. For system (2), under the condition (29), by small perturbations of the parameter group $\left(\delta, a_{30}, a_{12}\right.$, $\left.a_{32}, a_{14}, a_{34}, a_{16}, a_{33}, a_{15}, a_{61}, a_{05}, a_{43}, a_{51}, b_{33}\right)$, then there are 14 small amplitude limit cycles bifurcated from the origin.

\section{Acknowledgments}

This paper is partly supported by the Nature Science Foundation of China Grants 11261013 and 11361017 and the Nature Science Foundation of Guangxi (2012GXNSFAA053003). which is symmetric with respect to the $y$-axis, one has the following.

Theorem 10. The origin of system (2) is a center if and only if $\delta=0$ and (27) holds.

By $\lambda_{1}=\lambda_{2}=\cdots=\lambda_{13}=0, \lambda_{14} \neq 0$, one has the following.

Theorem 11. The origin of system (2) is a 14th-order weak focus if and only if

$$
\delta=a_{30}=a_{12}=a_{32}=a_{14}=a_{34}=0,
$$

$$
a_{61}=-\frac{1385}{64},
$$

$$
\begin{aligned}
& a_{05}=\frac{30075794600575314214479775}{606889200911167244345856}, \\
& a_{43}=-\frac{66625696625444520068811785}{303444600455583622172928},
\end{aligned}
$$$$
b_{33}^{2}=\frac{10913994716347225847247003725}{4779252457175442049223616},
$$

$$
a_{51}^{2}=\frac{1154557205782671354192175}{25287050037965301847744},
$$

$$
a_{16}=-\frac{3}{20} a_{51} b_{33}, \quad a_{33}=\frac{35}{8} a_{51}, \quad a_{15}=\frac{355}{128} a_{51} .
$$

By computing carefully, we obtain that the Jacobian determinant

\section{References}

[1] A. F. Andreev, "Investigation of the behaviour of the integral curves of a system of two differential equations in the neighbourhood of a singular point," American Mathematical Society Translations, vol. 8, pp. 183-207, 1958.

[2] V. V. Amel'kin, N. A. Lukashevich, and A. P. Sadovskiŭ, Nonlinear Oscillations in Second Order Systems, Belarusian State University, Minsk, Russia, 1982, (Russian).

[3] V. G. Romanovskii, "On the cyclicity of the equilibrium position of the center or focus type of a certain system," Vestnik St. Petersburg University: Mathematics, vol. 19, pp. 51-56, 1986.

[4] M. J. Álvarez and A. Gasull, "Monodromy and stability for nilpotent critical points," International Journal of Bifurcation and Chaos, vol. 15, no. 4, pp. 1253-1265, 2005. 
[5] A. F. Andreev, A. P. Sadovskiü, and V. A. Tsikalyuk, "The centerfocus problem for a system with homogeneous nonlinearities in the case of zero eigenvalues of the linear part," Differential Equations, vol. 39, no. 2, pp. 155-164, 2003.

[6] M. J. Álvarez and A. Gasull, "Generating limit cycles from a nilpotent critical point via normal forms," Journal of Mathematical Analysis and Applications, vol. 318, no. 1, pp. 271-287, 2006.

[7] A. Algaba, C. García, and M. Reyes, "Local bifurcation of limit cycles and integrability of a class of nilpotent systems of differential equations," Applied Mathematics and Computation, vol. 215, no. 1, pp. 314-323, 2009.

[8] Y. Liu and J. Li, "On third-order nilpotent critical points: integral factor method," International Journal of Bifurcation and Chaos, vol. 21, no. 5, pp. 1293-1309, 2011.

[9] M. Han and V. G. Romanovski, "Limit cycle bifurcations from a nilpotent focus or center of planar systems," Abstract and Applied Analysis, vol. 2012, Article ID 720830, 28 pages, 2012.

[10] Y. Liu and J. Li, "New study on the center problem and bifurcations of limit cycles for the Lyapunov system. I," International Journal of Bifurcation and Chaos, vol. 19, no. 11, pp. 3791-3801, 2009.

[11] Y. Liu and J. Li, "New study on the center problem and bifurcations of limit cycles for the Lyapunov system. II," International Journal of Bifurcation and Chaos, vol. 19, no. 9, pp. 3099-3807, 2009.

[12] Y. Liu and J. Li, "Bifurcations of limit cycles and center problem for a class of cubic nilpotent system," International Journal of Bifurcation and Chaos, vol. 20, no. 8, pp. 2579-2584, 2010.

[13] F. Li, Y. Liu, and Y. Wu, "Center conditions and bifurcation of limit cycles at three-order nilpotent critical point in a seventh degree Lyapunov system," Communications in Nonlinear Science and Numerical Simulation, vol. 16, no. 6, pp. 2598-2608, 2011.

[14] F. Li, Y. Liu, and H. Li, "Center conditions and bifurcation of limit cycles at three-order nilpotent critical point in a septic Lyapunov system," Mathematics and Computers in Simulation, vol. 81, no. 12, pp. 2595-2607, 2011. 


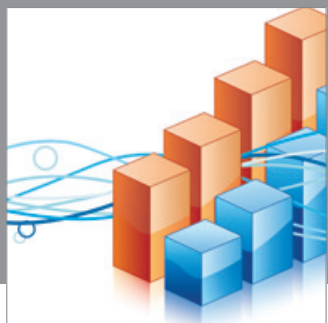

Advances in

Operations Research

mansans

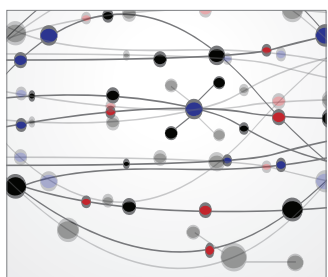

The Scientific World Journal
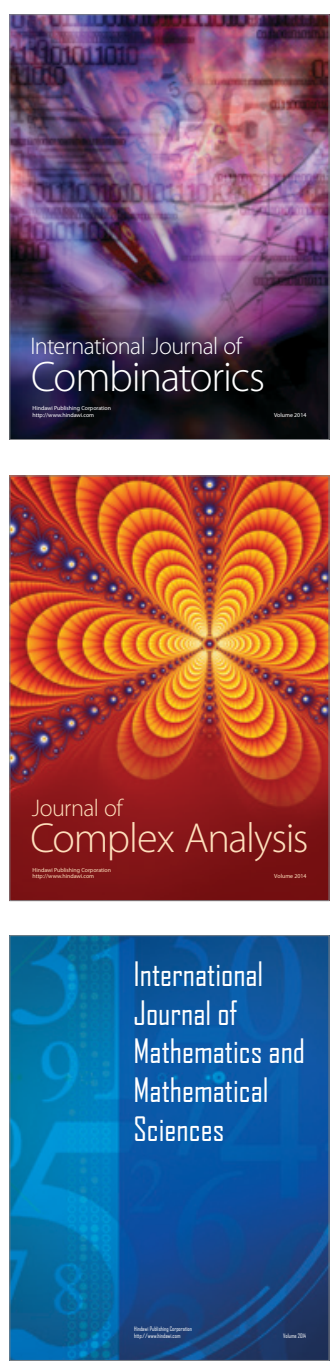
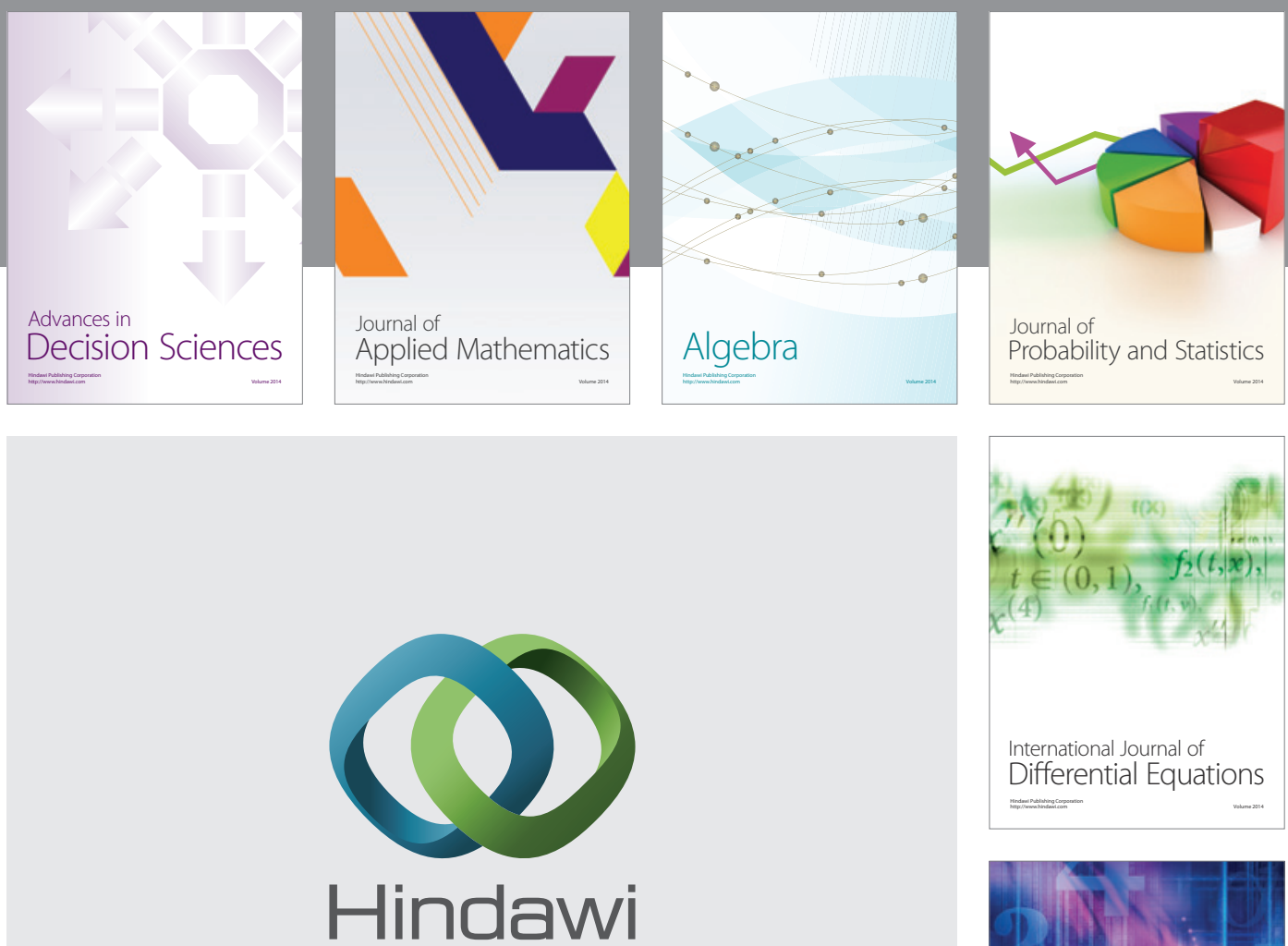

Submit your manuscripts at http://www.hindawi.com
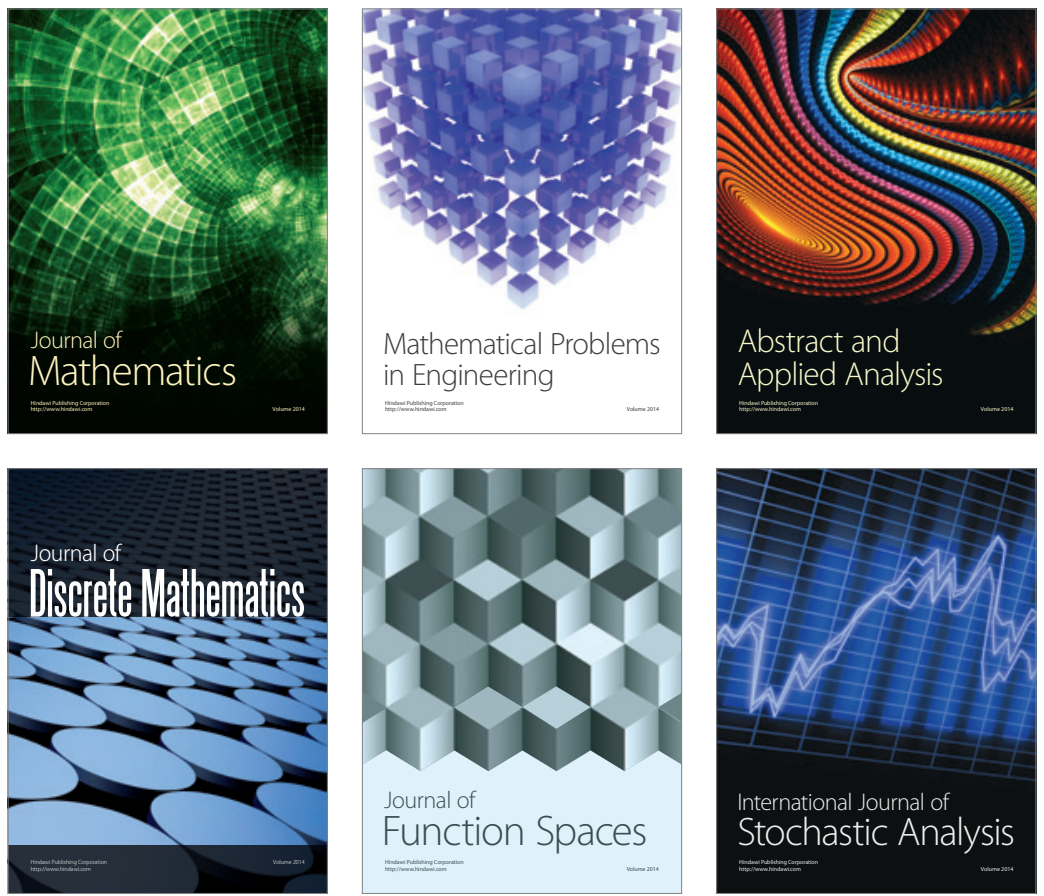

Journal of

Function Spaces

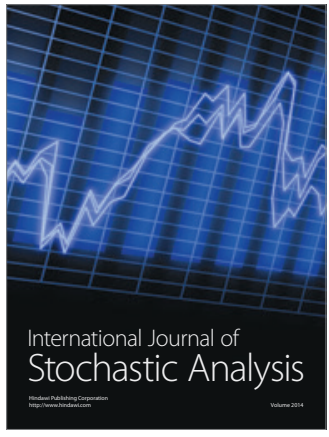

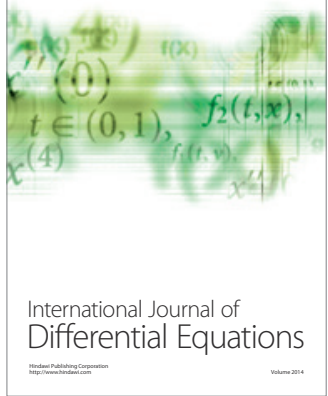
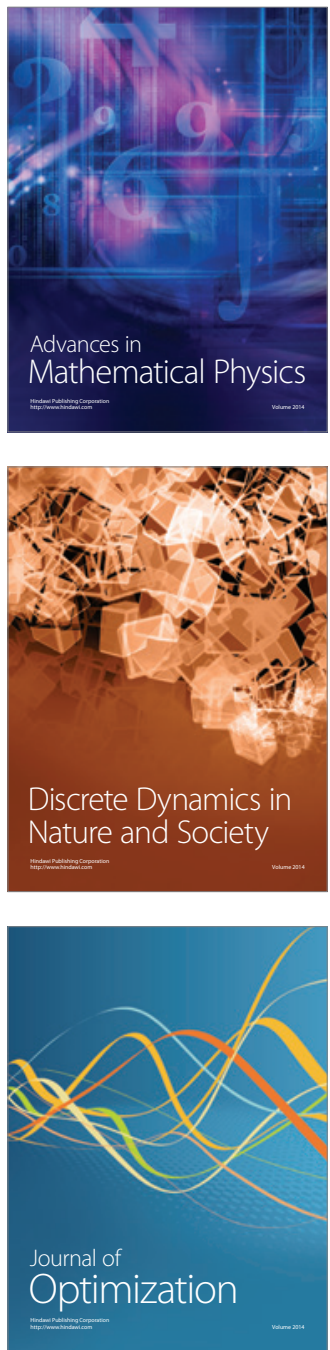